\title{
ORIGINAL
}

\author{
H. J. Böhm • G. A. Zickler • F. D. Fischer $1 D$ • J. Svoboda
}

\section{Strain and interface energy of ellipsoidal inclusions subjected to volumetric eigenstrains: shape factors}

Received: 23 June 2021 / Accepted: 21 October 2021 / Published online: 3 January 2022

(C) The Author(s) 2021

\begin{abstract}
Thermodynamic modeling of the development of non-spherical inclusions as precipitates in alloys is an important topic in computational materials science. The precipitates may have markedly different properties compared to the matrix. Both the elastic contrast and the misfit eigenstrain may yield a remarkable generation of elastic strain energy which immediately influences the kinetics of the developing precipitates. The relevant thermodynamic framework has been mostly based on spherical precipitates. However, the shapes of actual particles are often not spherical. The energetics of such precipitates can be met by adapting the spherical energy terms with shape factors. The well-established Eshelby framework is used to evaluate the elastic strain energy of inclusions with ellipsoidal shapes (described by the axes $a, b$, and $c$ ) that are subjected to a volumetric transformation strain. The outcome of the study is two shape factors, one for the elastic strain energy and the other for the interface energy. Both quantities are provided in the form of easy-to-use diagrams. Furthermore, threshold elastic contrasts yielding strain energy shape factors with the value 1.0 for any ellipsoidal shape are studied.
\end{abstract}

Keywords Precipitation $\cdot$ Misfit strain energy $\cdot$ Interface energy $\cdot$ Shape factors $\cdot$ Modeling thermodynamics

\section{Introduction and motivation}

The authors outlined in detail [1] how the strain energies of spheroidal inclusions can be obtained from the spherical case by shape factors. Understanding both the positive or negative roles of precipitates is an important topic in materials research; see, e.g., the pertinent chapter in the well-established book on kinetics of materials by Balluffi et al. [2] or exact engineering treatments such as [3]. The authors and associated researchers have provided in [4-7] essentially all kinetic relations, including also the mechanical terms, particularly the elastic strain energy contributions, derived for spherical inclusions. This established concept can now be adapted by the so-called shape factors in order to deal with non-spherical shapes of inclusions.

Supplementary Information The online version contains supplementary material available at https://doi.org/10.1007/ s00419-021-02066-1.

H. J. Böhm

Institute of Lightweight Design and Structural Biomechanics, Vienna University of Technology, 1040 Vienna, Austria

G. A. Zickler · F. D. Fischer $(\bowtie)$

Institute of Mechanics, Montanuniversitöl Leoben, 8700 Leoben, Austria

e-mail: mechanik@unileoben.ac.at

J. Svoboda

Institute of Physics of Materials, Academy of Sciences of the Czech Republic, 61662 Brno, Czech Republic 
Generally, one can observe that research on the behavior of transforming inclusions is still in progress; see, e.g., the recent work on circular (cylindrical) inclusions with a non-uniform eigenstrain $[8,9]$ in this journal. In the present study, particle shapes are represented by ellipsoids. This basic assumption offers two features:

- Three geometrical parameters, the principal axes $a, b, c$ of the ellipsoid, are available; the aspect ratio, $h$, is chosen as $h=c / \sqrt{a b}$ for compatibility with spheroidal shapes,

- The stress and strain fields in the inclusions are homogeneous, and the well-established "Eshelby concept" can be applied.

With respect to the above assumption, the following parameters are considered for a single inclusion embedded in an infinite matrix:

- The elastic properties $\left(E^{i}, v^{i}, E^{m}, v^{m}\right)$ of the inclusion (superscript " $i$ “") and the matrix (superscript " $m$ ") are each homogeneous and isotropic but, in general, different,

- The eigenstrain tensor is considered to be volumetric $\left(\tilde{\varepsilon}_{1}=\tilde{\varepsilon}_{2}=\tilde{\varepsilon}_{3}\right)$ and spatially constant in the inclusion.

We are aware of the problem that the interaction of several inclusions may perturb the uniform stress inside an inclusion to some extent. Here, we refer to the recent research by Schiavone et al.; see, e.g., [10].

\section{Strain energy density and Eshelby tensor}

As outlined in [1], the elastic strain energy density $V S E^{h}$ (i.e., the total elastic strain energy per unit volume) of a mutually non-interacting homogeneous inclusion $\left(\mathbf{E}^{i}=\mathbf{E}^{m}\right)$, or inhomogeneous inclusion $\left(\mathbf{E}^{i} \neq \mathbf{E}^{m}\right)$ (see Eq. (1)), subjected to a volumetric eigenstrain, can be expressed on the basis of the Eshelby framework as

$$
V S E^{h}=-\frac{1}{2} \tilde{\boldsymbol{\varepsilon}}^{T} \mathbf{E}^{m}(\mathbf{S}-\mathbf{I})\left[\left(\mathbf{E}^{i}-\mathbf{E}^{m}\right) \mathbf{S}+\mathbf{E}^{m}\right]^{-1} \mathbf{E}^{i} \tilde{\boldsymbol{\varepsilon}} .
$$

The superscript " $h$ " refers to the aspect ratio $h$. The tensors $\mathbf{E}^{m}$ and $\mathbf{E}^{i}$ stand for the elasticity tensors of the matrix and the inclusion, $\mathbf{S}$ for the Eshelby tensor, $\mathbf{I}$ for the unit tensor, and the vector $\tilde{\boldsymbol{\varepsilon}}$ for the volumetric eigenstrain. Restriction to the latter allows the use of a "reduced" $3 \times 3$ notation, compared to [1]. With suitable expressions for the Eshelby tensor, this approach holds for general ellipsoidal inclusions and for general elastic properties of matrix and inclusion.

Whereas the Eshelby tensors of spheroids, embedded in an isotropic matrix, can be given in closed form, for general ellipsoids they involve incomplete elliptic integrals of the first and second kinds (see the Appendix), which have to be evaluated numerically. For the present work, this was done by the efficient and accurate algorithms of Fukushima [11, 12]. As an alternative, surface integration in Fourier space by numerical quadrature [13] was implemented, allowing to handle matrices of general elastic symmetry.

\section{The shape factor $H(h)$ for the strain energy}

The shape factor for the strain energy density is defined as

$$
H(h)=V S E^{h} / V S E .
$$

Here, the quantity $V S E$ denotes the elastic strain energy density of a spherical inclusion with the same volume and the same elastic properties as the actual ellipsoidal inclusion as specified in Sect. 1.

Figure 1 shows the shape factor, obtained this way for a matrix Poisson ratio of $v^{m}=0.3$, and for inclusion Poisson ratios of $v^{i}=0.1,0.3$, and 0.4999 , and elastic contrasts, $\tilde{E}=E^{i} / E^{m}=1 / 3,1$, and 3. For each of these material combinations, $H(h)$ was evaluated as a function of the aspect ratio $h$ for the geometrical parameters $a / b=1,2$, and 3 . For these moderate elastic contrasts and geometrical parameters $a / b$, the results predicted for the ellipsoids are qualitatively similar to the ones for the corresponding spheroids, $a / b=1$; for details on the latter, see [1] (Figs. 1 and 2). The quantitative differences of the strain energies of spheroids and ellipsoids are also not significantly pronounced. The geometrical parameters $a / b$ give identical results as $b / a$. Accordingly, for this regime, the shape factors for the strain energy density of spheroidal inclusions given in [1] can be viewed as reasonable approximations for ellipsoids.

Qualitatively and quantitatively similar results on $H(h)$ were obtained for a nearly incompressible matrix, $v^{m}=0.49999$; see Fig. 2. All other parameters are set to be identical to those used in Fig. 1. 

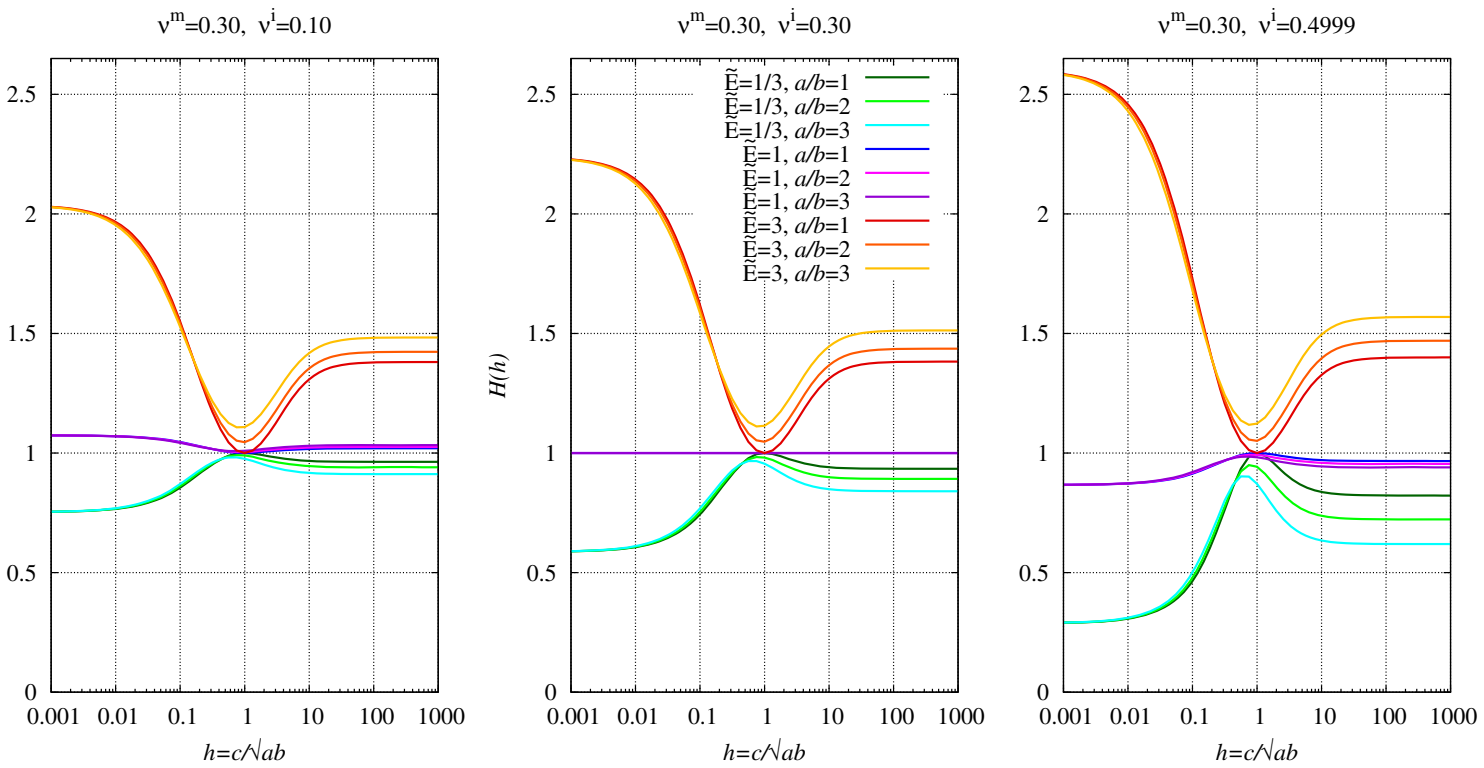

Fig. 1 Dependence of the shape factor $H(h)$ for elastic contrasts $\tilde{E}=1 / 3,1,3, v^{m}=0.3$ and geometrical parameters $a / b=1$, 2, 3 for $v^{i}=0.1$ (left), 0.3 (center), and 0.49999 (right)
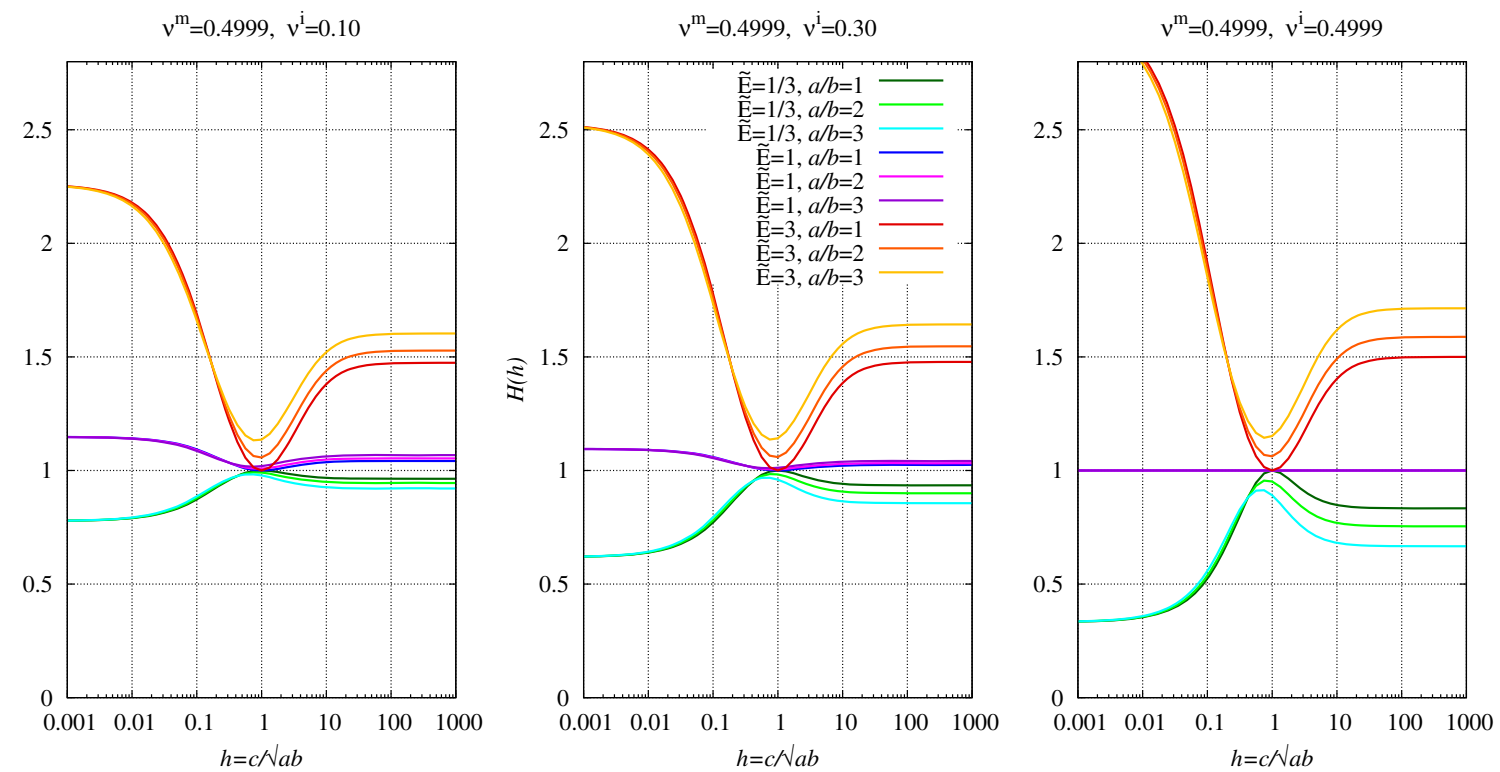

Fig. 2 Dependence of the shape factor $H(h)$ for elastic contrasts $\tilde{E}=1 / 3,1,3, v^{m}=0.49999$ and geometrical parameters $a / b$ $=1,2,3$ for $v^{i}=0.1$ (left), 0.3 (center), and 0.49999 (right)

Three types of behavior of $H(h)$ are evident in Figs. 1 and 2: The red, yellow, and orange curves pertaining to $\tilde{E}=3$ show absolute minima $H_{\text {min }} \geq 1$ at or slightly below $h=1$. The green and cyan curves describe cases with $\tilde{E}=1 / 3$ and show absolute maxima $H_{\max } \leq 1$. However, the blue and purple curves in Fig. 1 (center) and Fig. 2 (right), i.e., the curves pertaining to homogeneous inclusions, are constant with $H=1$. Such a behavior was previously noted for spheroidal inclusions in [1], where homogeneous and inhomogeneous configurations with $H=1$ were found to be associated with threshold elastic contrasts $\tilde{E}_{t h}$. The latter quantities, which follow Eq. (3), were evaluated numerically and are shown in Fig. 3 in [1]. The presence of a minimum of $H(h)$ is indicated by $\tilde{E}>\tilde{E}_{t h}$ and that of a maximum by $\tilde{E}<\tilde{E}_{t h}$. The rather simple expression

$$
\tilde{E}_{t h}=\left(1+v^{i}\right) /\left(1+v^{m}\right)
$$



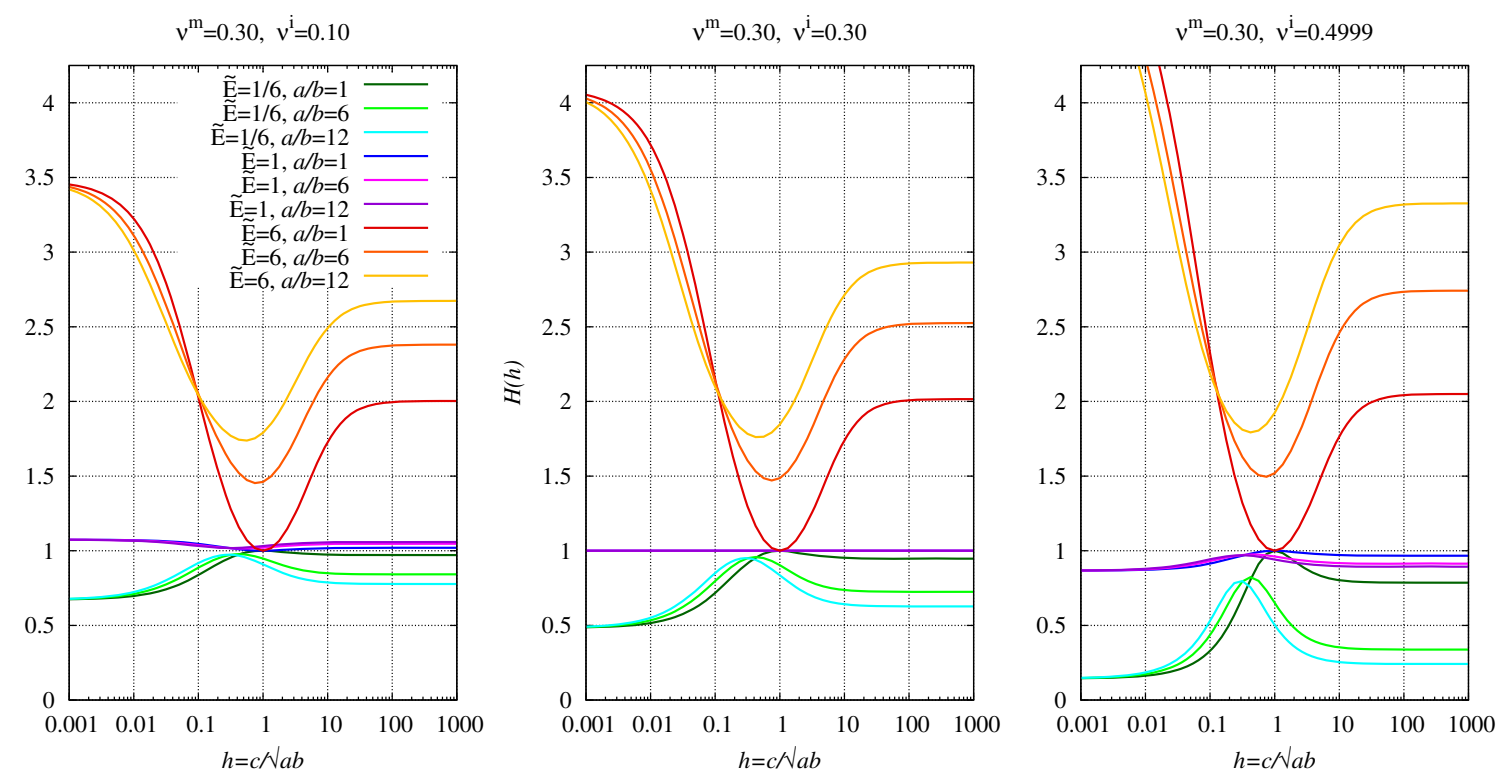

Fig. 3 Dependence of the shape factor $H(h)$ for elastic contrasts $\tilde{E}=1 / 6,1,6, v^{m}=0.3$ and geometrical parameters $a / b=1$, 6,12 for $v^{i}=0.1$ (left), 0.3 (center), and 0.49999 (right)

can be obtained for the threshold contrast of spheroidal inhomogeneities by requiring that the sum of the matrix elements of the top left $3 \times 3$ submatrix of the inverse term in square brackets in Eq. (1) is independent of $a$, $b$, and $c$. Equation (3) implies that $\tilde{E}_{t h}$ are associated with configurations where matrix and precipitates have identical shear moduli, $G^{i}=G^{m}$. Homogeneous inclusions giving $H(h)=1$, [14], are a special case of Eq. (3). Numerical experiments strongly indicate that Eq. (3) also holds for general ellipsoids.

If the elastic contrast $\tilde{E}$ is increased and/or the ellipsoidal shapes differ more strongly from spheroids, the shape factors $H(h)$ for ellipsoids deviate in a more pronounced way from the results obtained for spheroids. This is evident in Fig. 3, where the elastic contrasts take values of $E^{i} / E^{m}=1 / 6,1$, and 6 and the geometrical parameters are chosen as $a / b=1,6$, and 12 . The role of the threshold elastic contrasts, $\tilde{E}_{t h}$, is not affected by such benign conditions. However, on the one hand, the minima of $H(h)$ for $\tilde{E}>\tilde{E}_{t h}$ markedly exceed the value of unity associated with spheroids, and the maxima of $H(h)$ for $\tilde{E}<\tilde{E}_{t h}$ fall clearly below unity. On the other hand, these minima and maxima are shifted to aspect ratios $h<1$. For such cases, the shape factors of the spheroids can no longer serve as approximations and, therefore, Eqs. (1) and (2) must be evaluated with the appropriate parameters.

\section{The shape factor $F(h)$ for the interface energy}

The shape factor for the interface energy of an ellipsoidal inclusion is given by $F(h)=A^{h} / A$, where $A^{h}$ represents the surface area of the ellipsoid and $A$ that of a sphere with the same volume as the ellipsoid.

A closed expression for the surface of an ellipsoid does not exist. However, $A^{h}$ is very well approximated by $4 \pi\left[\frac{(a b)^{n}+(a c)^{n}+(b c)^{n}}{3}\right]^{\frac{1}{n}}$ with $n=8 / 5$, known as Knud Thomsen's formula [15]. The resulting behavior of $F(h)$ is displayed in Fig. 4 with $\varepsilon=1-b / a, b \leq a$ as parameter.

In order to facilitate obtaining estimates of $H(h)$ and $F(h)$ for given values of $\tilde{E}, v^{i}$ and $a / b$ by interpolation, high-resolution versions of Figs. 1, 2, 3, and 4 are provided as supplementary information.

\section{Conclusion}

The role of mechanical energy terms has become increasingly important in modeling evolving microstructures, compared to, for example, the application of the microscopic phase-field model [16]. The present contribution 


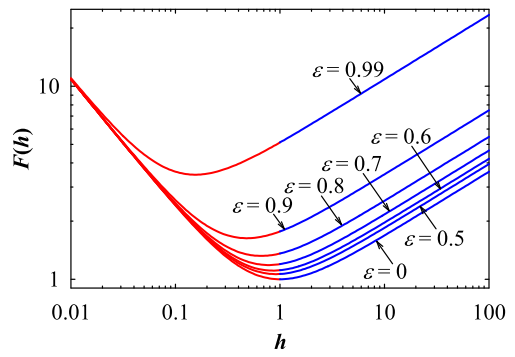

Fig. 4 Dependence of the shape factor of $F(h)$ on the aspect ratio $h$ and the parameter $\varepsilon=1-b / a$

provides diagrams that allow accounting for the effects of the inclusion shape on both the elastic strain energy and the surface energy. On this basis, the mechanical part of the Gibbs energy, required for modeling the transformation kinetics, can be evaluated more accurately than previously, when simplified approximations had to be used.

Acknowledgements F.D.F. and J.S. appreciate the financial support by the Austrian Federal government (in particular from the Bundesministerium für Verkehr, Innovation and Technologie and the Bundesministerium für Wirtschaft und Arbeit) and the Styrian Provincial Government, represented by Österreichische Forschungsförderungsgesellschaft mbH and by Steirische Wirtschaftsförderungsgesellschaft $\mathrm{mbH}$, within the research activities of the K2 Competence Centre on "Integrated Research in Materials, Processing and Product Engineering," operated by the Materials Center Leoben Forschung GmbH in the framework of the Austrian COMET Competence Centre Programme, Project A1.23.

Funding Open access funding provided by Montanuniversität Leoben. J.S. gratefully acknowledges the financial support provided by the Ministry of Education and Sports of the Czech Republic within the research project "Architectured materials designed for additive manufacturing," Reg. No.: CZ.02.1.01/0.0/0.0/16 025/0007304.

\section{Declaration}

Conflict of interest The authors declare that they have no known competing financial interests or personal relationships that could have appeared to influence the work reported in this paper.

Open Access This article is licensed under a Creative Commons Attribution 4.0 International License, which permits use, sharing, adaptation, distribution and reproduction in any medium or format, as long as you give appropriate credit to the original author(s) and the source, provide a link to the Creative Commons licence, and indicate if changes were made. The images or other third party material in this article are included in the article's Creative Commons licence, unless indicated otherwise in a credit line to the material. If material is not included in the article's Creative Commons licence and your intended use is not permitted by statutory regulation or exceeds the permitted use, you will need to obtain permission directly from the copyright holder. To view a copy of this licence, visit http://creativecommons.org/licenses/by/4.0/.

\section{Appendix}

\section{Eshelby tensor for ellipsoidal inclusions}

Within the "reduced" $3 \times 3$ notation sufficient for handling volumetric strains, the components of the Eshelby tensor $\mathbf{S}$ for general ellipsoidal inclusions, required for evaluating Eq. (1), can be adapted to the present notation from [14] to take the form

$$
\begin{aligned}
& S(1,1)=\frac{1}{8 \pi\left(1-v^{m}\right)}\left[3 c^{2} I_{11}+\left(1-2 v^{m}\right) I_{1}\right] \\
& S(1,2)=\frac{1}{8 \pi\left(1-v^{m}\right)}\left[a^{2} I_{12}+\left(1-2 v^{m}\right) I_{1}\right] \\
& S(1,3)=\frac{1}{8 \pi\left(1-v^{m}\right)}\left[b^{2} I_{13}+\left(1-2 v^{m}\right) I_{1}\right]
\end{aligned}
$$




$$
\begin{aligned}
& S(2,1)=\frac{1}{8 \pi\left(1-v^{m}\right)}\left[c^{2} I_{12}+\left(1-2 v^{m}\right) I_{2}\right] \\
& S(2,2)=\frac{1}{8 \pi\left(1-v^{m}\right)}\left[3 a^{2} I_{22}+\left(1-2 v^{m}\right) I_{2}\right] \\
& S(2,3)=\frac{1}{8 \pi\left(1-v^{m}\right)}\left[b^{2} I_{23}+\left(1-2 v^{m}\right) I_{2}\right] \\
& S(3,1)=\frac{1}{8 \pi\left(1-v^{m}\right)}\left[c^{2} I_{13}+\left(1-2 v^{m}\right) I_{3}\right] \\
& S(3,2)=\frac{1}{8 \pi\left(1-v^{m}\right)}\left[a^{2} I_{23}+\left(1-2 v^{m}\right) I_{3}\right] \\
& S(3,3)=\frac{1}{8 \pi\left(1-v^{m}\right)}\left[3 b^{2} I_{33}+\left(1-2 v^{m}\right) I_{3}\right]
\end{aligned}
$$

The quantity $v^{m}$ stands for the Poisson ratio of the matrix. Equation (A1) uses the assumption $c>a>b$, and the 1-direction is associated with $c$, the 2-direction with $a$ and the 3-direction with $b$. The terms $I_{j}$ and $I_{j k}$ are defined as

$$
\begin{aligned}
& I_{1}=\frac{4 \pi a b c}{\left(c^{2}-a^{2}\right) \sqrt{c^{2}-b^{2}}}[F(\theta, k)-E(\theta, k)] \\
& I_{2}=I_{1}-I_{3} \\
& I_{3}=\frac{4 \pi a b c}{\left(a^{2}-b^{2}\right) \sqrt{c^{2}-b^{2}}}\left[\frac{a \sqrt{c^{2}-b^{2}}}{c b}-E(\theta, k)\right] \\
& I_{11}=\frac{1}{3}\left(\frac{4 \pi}{c^{2}}-I_{12}-I_{13}\right) \\
& \left.I_{12}=\left(I_{2}-I_{1}\right) / c^{2}-a^{2}\right) \\
& \left.I_{13}=\left(I_{3}-I_{1}\right) / c^{2}-b^{2}\right) \\
& I_{22}=\frac{1}{3}\left(\frac{4 \pi}{a^{2}}-I_{12}-I_{23}\right) \\
& I_{23}=\left(I_{3}-I_{2}\right) /\left(a^{2}-b^{2}\right) \\
& I_{33}=\frac{1}{3}\left(\frac{4 \pi}{b^{2}}-I_{13}-I_{23}\right)
\end{aligned}
$$

$E(\theta, k)$ and $F(\theta, k)$ are the incomplete elliptic integrals of the first and second kinds, defined as

$$
\begin{aligned}
& E(\theta, k)=\int_{0}^{\theta}\left(1-k^{2} \sin ^{2} \omega\right)^{-1 / 2} d \omega \\
& F(\theta, k)=\int_{0}^{\theta}\left(1-k^{2} \sin ^{2} \omega\right)^{1 / 2} d \omega
\end{aligned}
$$

with

$$
\begin{aligned}
& \theta=\sin ^{-1}\left(\sqrt{1-\frac{b^{2}}{c^{2}}}\right) \\
& k^{2}=\frac{c^{2}-a^{2}}{c^{2}-b^{2}}
\end{aligned}
$$

\section{References}

1. Böhm, H.J., Zickler, G.A., Fischer, F.D., Svoboda, J.: Role of elastic strain energy in spheroidal precipitates revisited. Mech. Mater. 155, 103781 (2021) 
2. Balluffi, R.W., Allen, S.M., Cater, W.C.: Kinetics of Materials. Wiley, Hoboken (2005)

3. Amstutz, H., Vormwald, M.: Elastic spherical inhomogeneity in an infinite elastic solid: an exact analysis by an engineering treatment of the problem based on the corresponding cavity solution. Arch. Appl. Mech. 91, 1577-1603 (2021)

4. Svoboda, J., Fischer, F.D., Fratzl, P., Kozeschnik, E.: Modelling of kinetics in multi-component multi-phase systems with spherical precipitates I. - Theory. Mat. Sci. Eng. A 385, 166-174 (2004)

5. Kozeschnik, E., Svoboda, J., Fratzl, P., Fischer, F.D.: Modelling of kinetics in multi-component multi-phase multi-particle systems with spherical precipitates II. - Numerical solution and application. Mat. Sci. Eng. A 385, 157-165 (2004)

6. Kozeschnik, E., Svoboda, J., Fischer, F.D.: Shape factors in modeling of precipitation. Mat. Sci. Eng. A 441, 68-72 (2006)

7. Svoboda, J., Fischer, F.D., Mayrhofer, P.H.: A model for evolution of shape changing precipitates in multicomponent systems. Acta mater. 56, 4896-4904 (2008)

8. Yu, X.-W., Wang, Z.-W., Wang, H., Leng, N.-Y.: Eshelby's circular cylindrical inclusion with polynomial eigenstrains in transverse direction by residue theorem. Arch. Appl. Mech. 91, 1437-1447 (2021)

9. Wang, B., Zhao, W., Ma, L.: Analysis of a finite matrix with an inhomogeneous circular inclusion subjected to a non-uniform eigenstrain. Arch. Appl. Mech. 90, 945-956 (2020)

10. Dai, M., Schiavone, P.: Need the uniform stress field inside multiple interacting inclusions be hydrostatic. J. Elasticity 143, 195-207 (2021)

11. Fukushima, T.: Fast computation of incomplete elliptic integral of first kind by half argument transformation. Numer. Math. 116, 687-719 (2010)

12. Fukushima, T.: Precise and fast computation of a general incomplete elliptic integral of second kind by half and double argument transformations. J. Comput. Appl. Math. 235, 4140-4148 (2010)

13. Gavazzi, A.C., Lagoudas, D.C.: On the numerical evaluation of Eshelby's tensor and its application to elastoplastic fibrous composites. Comput. Mech. 7, 12-19 (1990)

14. Mura, T.: Micromechanics of Defects in Solids. Martinus Nijhoff Publishers, Dordrecht (1987)

15. Thomsen K. (2004). http://www.numericana.com/answer/ellipsoid.htm\#thomsen.

16. Jeong D., Lee S., Kim J. (2015). An efficient numerical method for evolving microstructures with strong elastic inhomogeneity. Model. Sim. Mater. Sci. Eng. 23, 045007.

Publisher's Note Springer Nature remains neutral with regard to jurisdictional claims in published maps and institutional affiliations. 